\title{
Toxicidad por colistina: hiperpigmentación cutánea, neurotoxicidad y nefrotoxicidad. Reporte de caso
}

\author{
Colistin toxicity: skin hyperpigmentation, neuro and renal toxicity. A case report
}

Diana Fernández Merjildo ${ }^{1, a}$, Candy Caytano García ${ }^{1, b}$, Aldo Gálvez Canseco ${ }^{2, c}$, Coralith García Apac ${ }^{2, d}$

\section{RESUMEN}

La colistina o polimixina $\mathrm{E}$ es un antibiótico cuyo uso fue descontinuado por la toxicidad renal y neurológica relacionadas al uso de colistina sulfato. Estos efectos adversos han disminuido con el uso del profármaco colistimetato sódico. Actualmente el uso de colistina es más frecuente debido al incremento de infecciones ocasionadas por bacilos Gram negativos multirresistentes, sobre todo en las unidades de cuidados intensivos. Presentamos el caso de una mujer de 50 años de edad con antecedente de consumo de anti-inflamatorios no esteroideos y corticoides, posoperada de perforación gástrica que evolucionó con colecciones abdominales por Acinetobacter sp. multirresistente. Recibió 34 días de colistina endovenosa y desarrolló hiperpigmentación cutánea, ataxia (neurotoxicidad) y falla renal (nefrotoxicidad) de forma secuencial secundaria a la administración de colistina, los efectos adversos desaparecieron con la suspensión del antibiótico.

PALABRAS CLAVE: Colistina, riñón/toxicidad, síndromes de neurotoxicidad, hiperpigmentación. (Fuente: DeCS BIREME).

\section{SUMMARY}

Colistin or polymyxin $\mathrm{E}$ is an antibiotic that was discontinued due to its renal and neurologic toxicity related to its colistin sulfate content. These adverse effects have been reduced with the use of sodium colistemathe. There is currently a more frequent use of colistin due to an increase of multi-resistant Gram negative infections, particularly in intensive care units. We present the case of a 50-year-old woman with history of surgery due to gastric perforation, use of steroids and non-steroidal anti-inflammatory drugs, that developed intra-abdominal abscesses due to multidrug resistant Acinetobacter spp. She received 34 days of intravenous colistin and developed skin hyperpigmentation, ataxia and renal failure. These adverse effects disappeared with discontinuation of the drug.

KEYWORDS: Colistin, kidney/toxicity, neurotoxicity syndromes, hyperpigmentation. (Source: MeSH NLM).

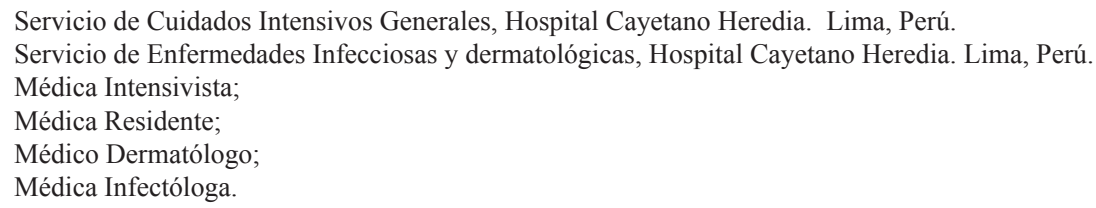




\section{INTRODUCCIÓN}

La colistina o polimixina $E$ es un antibiótico producido por cepas de la bacteria Paenibacillus polymyxa variedad Colistinus, cuyo uso fue abandonado en la década de 1970 debido a su toxicidad renal y neurológica; actualmente su uso es más frecuente debido infecciones ocasionadas por bacilos Gram negativos (Acinetobacter y Pseudomonas aeruginosa) multirresistentes (MDR) sobre todo en las unidades de cuidados intensivos (UCI) (1).

La toxicidad por colistina fue relacionada al uso de colistina sulfato, los eventos adversos como toxicidad renal y neurológica han disminuido con el uso del profármaco colistimetato sódico (2); la dosis acumulada total de colistina, hipoalbuminemia, estados de shock y el uso concomitante de fármacos potencialmente nefrotóxicos incrementan el riesgo de nefrotoxicidad; sin embargo, la toxicidad neurológica es menos frecuente (3).

Debido al creciente incremento de infecciones por gérmenes MDR, colistina es un antimicrobiano cada vez más utilizado; por lo que, el reconocimiento de los eventos adversos graves relacionados con su uso, son de vital importancia. Presentamos un caso de triple toxicidad por colistina manifestado por hiperpigmentación cutánea, ataxia (neurotoxicidad) y falla renal oligúrica con requerimiento de hemodiálisis (nefrotoxicidad) en una paciente con infección abdominal por Acinetobacter sp. MDR.

\section{PRESENTACIÓN DEL CASO}

Mujer de 50 años de edad, quien 6 meses antes del ingreso por Emergencia inició tratamiento

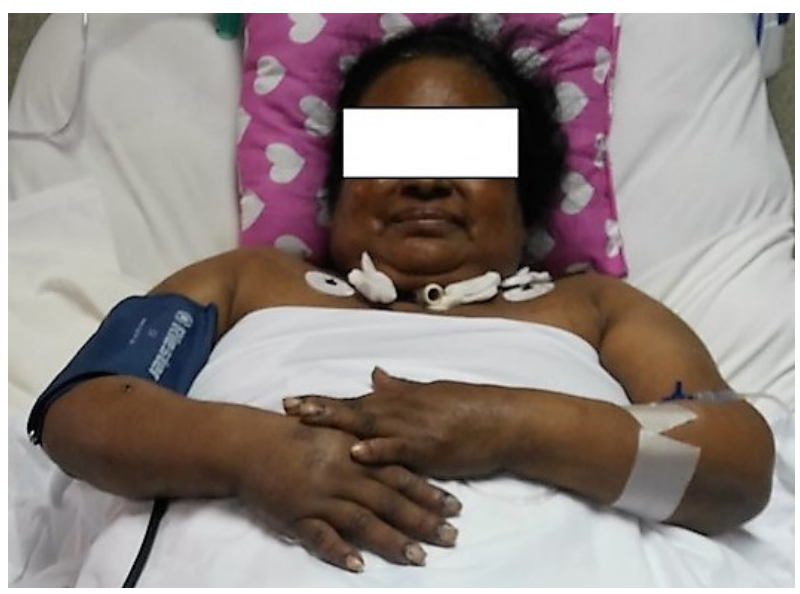

Figura 1. Hiperpigmentación cutánea, 7 días después de la administración de colistina. farmacológico con anti-inflamatorios no esteroideos (AINE) y corticoides por antecedente de lumbago. Acudió por dolor abdominal intenso, con signos de irritación peritoneal, por lo que fue intervenida quirúrgicamente por el diagnóstico de abdomen agudo quirúrgico; los hallazgos operatorios fueron perforación gástrica en cara anterior del píloro $(2 \times 1 \mathrm{~cm})$ y $1500 \mathrm{ml}$ de líquido intestinal en cavidad abdominal, le realizaron lavado de cavidad abdominal y colocación de parche de epiplón más drenajes.

En el posoperatorio inmediato, ingresó a la unidad de cuidados intensivos (UCI) con presión arterial $105 / 70 \mathrm{~mm} \mathrm{Hg}$ y soporte vasopresor (noradrenalina a $0,5 \mathrm{ug} / \mathrm{kg} / \mathrm{min}$ ), frecuencia cardiaca 132 latidos por minuto, intubada y conectada a ventilador mecánico con saturación por pulsioximetria $100 \%$ a una fracción inspirada de oxigeno de $50 \%$. Los exámenes de laboratorio revelaron: hemoglobina $8,3 \mathrm{~g} / \mathrm{dl}$, cuenta de leucocitos $15540 / \mathrm{mm}^{3}$, plaquetas $162000 / \mathrm{mm}^{3}$, INR 3,81, glucosa sérica $135 \mathrm{mg} / \mathrm{dl}$, creatinina 0,4 $\mathrm{mg} / \mathrm{dl}$, urea $48,3 \mathrm{mg} / \mathrm{dl}$, sodio $150 \mathrm{mmol} / 1$, potasio $3,18 \mathrm{mmol} / \mathrm{l}$, bilirrubina total $1,0 \mathrm{mg} / \mathrm{dl}$, TGO 496 U/1, TGP $571 \mathrm{U} / 1$, fosfatasa alcalina $84 \mathrm{UI} / 1$, gamaglutamil transpeptidasa $46 \mathrm{UI} / 1$, albúmina $2,1 \mathrm{~g} / \mathrm{dl}$; la gasometría arterial, $\mathrm{pH} 7,24, \mathrm{pCO}_{2} 46,4 \mathrm{~mm} \mathrm{Hg}, \mathrm{p} 0_{2}$ $152 \mathrm{~mm} \mathrm{Hg}, \mathrm{HCO}_{3} 18 \mathrm{mmol} / \mathrm{l}, \mathrm{SO}_{2} 99,9 \%$ y lactato $6,4 \mathrm{mmol} / 1$; la puntuación SOFA (Score de evaluación secuencial de falla de órgano) fue 7 puntos (mortalidad estimada $15-20 \%$ ).

A 4 días de su ingreso a la UCI fue re-intervenida quirúrgicamente por sospecha de dehiscencia del parche de epiplón, hallándose un plastrón a nivel del

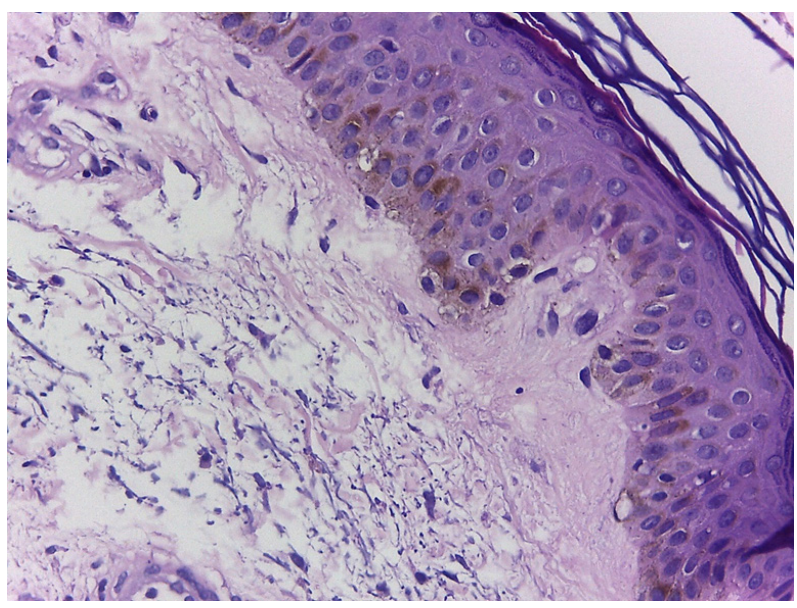

Figura 2. Biopsia de piel obtenida del antebrazo de la paciente con tinción de hematoxilina-eosina

(100X). Hallazgos histológicos relacionados a hiperpigmentación difusa (epidermis con incremento del pigmento en los queratinocitos). 
parche conformado por epiplón, asas intestinales, colón e hígado y liquido purulento en el espacio subfrénico izquierdo y en fondo de saco de Douglas. Se administró nutrición mixta (parenteral y enteral). Evolucionó febril y en la tomografía de abdomen se evidenciaron múltiples colecciones intra-abdominales que fueron drenadas por vía percutánea; en el cultivo se aisló Acinetobacter sp. MDR, por lo que, inició colistina endovenosa (EV) $150 \mathrm{mg}$ dos veces por día.

Seis días después del inicio de colistina EV, se agregó fluconazol EV a $200 \mathrm{mg}$ cada 12 horas por persistencia de la fiebre y sospecha de candidemia (Cándida Score: 4 puntos) y tres días después vancomicina EV a $15 \mathrm{mg} / \mathrm{kg}$ cada 12 horas por bacteriemia por Staphylococcus aureus meticilino resistente; fluconazol fue administrado por 23 días y vancomicina por 14 días; el hemocultivo para hongos fue negativo.

Siete días después del inicio de colistina la paciente presentó hiperpigmentación cutánea a predominio facial (Figura 1), bajo la sospecha de hiperpigmentación inducida por toxicidad a colistina, se solicitaron exámenes auxiliares para excluir otras causas de hiperpigmentación como insuficiencia suprarrenal aguda (por supresión de corticoides) y deficiencias nutricionales. El cortisol sérico a las $8.00 \mathrm{am}$, fue 20,61 ug/dl (VN: 1,9 - 22,3 ug/dl), descartándose enfermedad de Addison. La biopsia de piel por sacabocado, mostró como único hallazgo histológico una epidermis con incremento del pigmento en los queratinocitos. No se evidenció infiltrado inflamatorio ni incontinencia pigmentaria en la dermis (Figura 2).

Al término del tratamiento con vancomicina y fluconazol y aun recibiendo colistina se observó elevación de la creatinina $(2,3 \mathrm{mg} / \mathrm{dl})$ y urea sérica $(91 \mathrm{mg} / \mathrm{dl})$, además ataxia en extremidades superiores; para excluir como causa el déficit de vitamina $\mathrm{B}_{12}$ se realizó una medición sérica, cuyo valor fue normal 274,4 pg/ml (VN: 228 - $1515 \mathrm{pg} / \mathrm{ml}$ ); días posteriores progresó con oliguria y mayor incremento de creatinina $(7,7 \mathrm{mg} / \mathrm{dl})$ y urea sérica $(276 \mathrm{mg} / \mathrm{dl})$. Ante la sospecha de toxicidad por colistina se suspendió el antibiótico y se inició hemodiálisis por falla renal aguda, recuperándose la función renal (Gráfico 1) y disminución de la hiperpigmentación facial tres semanas después.

\section{DISCUSIÓN}

La hiperpigmentación cutánea en adultos secundario a la administración de polimixina $\mathrm{B}$ ha sido descrito en algunos reportes de casos (4), siendo menos frecuente en pacientes pediátricos (5). El mecanismo fisiopatológico no está completamente elucidado, sin embargo, un mecanismo propuesto es el incremento de la melanogénesis por mediadores inflamatorios

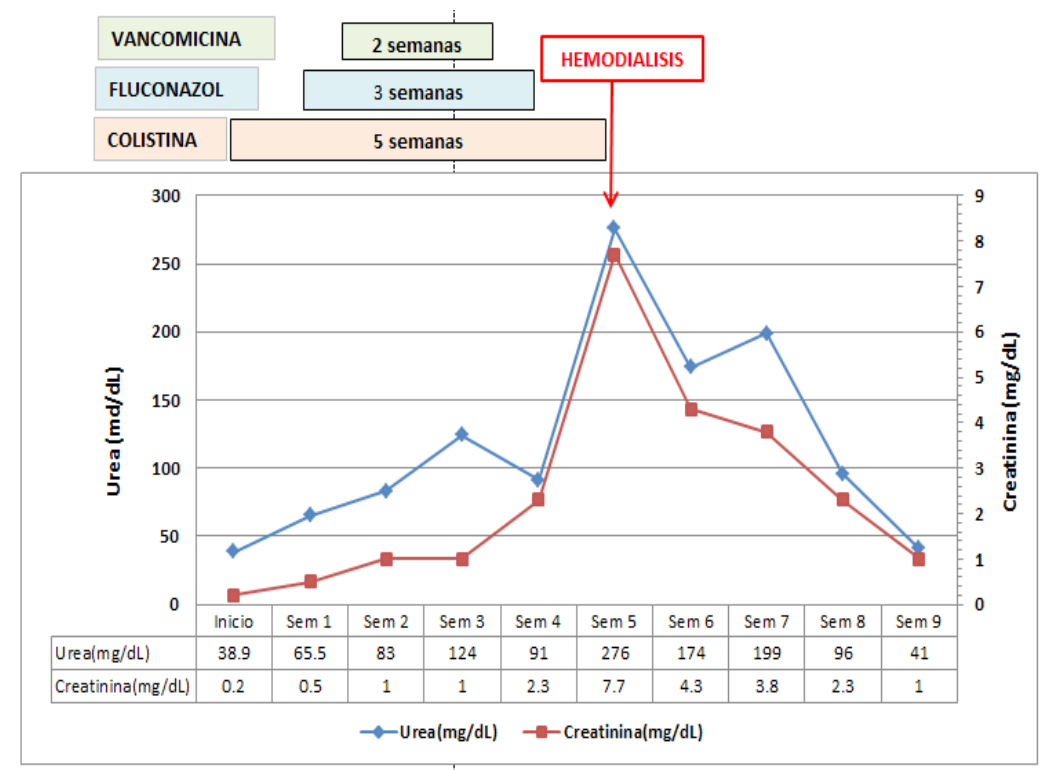

Gráfico 1. Niveles de urea y creatinina sérica, y su relación con la administración de Vancomicina, fluconazol y colistina. Fluconazol fue iniciado 6 días después de colistina, vancomicina 9 días después de colistina. 
como la histamina, cuya liberación es incrementada por las polimixinas; el incremento de la pigmentación cutánea es mayor en áreas de exposición como la región facial y extremidades debido a la densidad elevada de melanocitos en dichas áreas (6).

En el caso presentado, el incremento de la pigmentación fue a predominio facial, los hallazgos histológicos confirmaron el incremento de la melanogénesis por la presencia elevada del pigmento restringido a la epidermis. La hiperpigmentación cutánea difusa está asociada principalmente a la enfermedad de Addison, la deficiencia de vitamina $\mathrm{B}_{12}$ y el uso de fármacos; los niveles de cortisol y vitamina $\mathrm{B}_{12}$ séricos normales alejaron la posibilidad etiológica, mientras que la suspensión de colistina se asoció con la resolución del cuadro clínico dermatológico (7).

Se consideró que la ataxia que presentó la paciente fue secundaria a toxicidad neurológica por colistina en ausencia de hipovitaminosis (niveles séricos normales de Vitamina $\mathrm{B}_{12}$ ). Se ha observado que, la interacción de colistina con las células neuronales está relacionado con el desarrollo de parestesias periféricas y orofaciales, trastornos visuales, vértigo, confusión mental, ataxia y convulsiones (8). Se reportaron casos esporádicos de apnea en pacientes con insuficiencia renal crónica, que recibieron colistina por vía intramuscular o fármacos que potencialmente inducen debilidad de los músculos respiratorios (9).

La toxicidad renal es el efecto adverso más frecuente debido a que la colistina tiene excreción renal, en el caso presentado se consideró que el principal desencadenante de nefrotoxicidad fue la administración de colistina (dosis acumulada total), sin embargo, la administración concomitante de vancomicina y fluconazol, la disfunción renal previa y la hipoalbuminemia pudieron generar un efecto sinérgico. Estudios electrofisiológicos in vitro han demostrado que la exposición prolongada al antibiótico está relacionada con toxicidad urotelial por incremento de la conducción transepitelial (10).

El uso de colistina alcanza tasas de nefrotoxicidad de hasta $53,5 \%$, se ha evidenciado que la incidencia es 2,5 a 7 veces mayor en pacientes con disfunción renal y puede incrementar en presencia de factores predisponentes (enfermedad renal crónica, uso de drogas nefrotóxicas e hipovolemia) (11); los casos de neurotoxicidad secundario a colistina son raros, tasas actuales estiman una frecuencia entre 0 y $7 \%$ (12).
El incremento de infecciones por gérmenes MDR ha desencadenado el aumento del uso de colistina, en pacientes críticamente enfermos con falla multiorgánica, la falla renal puede alterar la farmacocinética de la colistina, por lo que, la modificación de la dosis es imprescindible para lograr máxima eficacia y toxicidad mínima (13). Aunque la neurotoxicidad es rara y la nefrotoxicidad es reversible ensombrece el pronóstico, por lo que, monitorizar la función renal y evitar el uso concomitante de fármacos nefrotóxicos o la administración por tiempos prolongados para disminuir los efectos adversos.

Una limitante fue la falta de medición de los niveles séricos de colistina debido a que no se encuentra disponible en nuestro país; sin embargo, la resolución de la pigmentación cutánea, la ataxia y de la disfunción renal una vez suspendida la administración de colistina más los hallazgos histológicos confirmaron la causalidad de los eventos tóxicos secundarios a colistina.

\section{Declaración de financiamiento y de conflictos de interés:}

El reporte fue financiado por los autores, y declaran que no existe relación, condición o circunstancia que pueda reducir la objetividad en la interpretación del artículo.

\section{Contribución de autoría:}

DFM: Concepción, redacción, revisión crítica y aprobación de la versión final del artículo; CCG: Redacción y revisión crítica del artículo; AGC: Asesoría técnica mediante la confirmación diagnóstica por patología y generación de imágenes; CGA: Revisión crítica y aprobación de la versión fina.

\section{Correspondencia:}

Diana Fernández Merjildo

Correo electrónico: diana.fernandez.m@upch.pe

\section{REFERENCIAS BIBLIOGRÁFICAS}

1. Briceño DF, Quinn JP, Villegas MV. Treatment options for multidrug-resistant nonfermenters. Expert Rev Anti Infect Ther. 2010; 8(3):303-15.

2. Falagas ME, Kasiakou SK. Toxicity of polymyxins: a systematic review of the evidence from old and recent studies. Crit Care. 2006; 10:R27.

3. Spapen H, Jacobs R, Van Gorp V, Troubleyn J, Honoré 
PM. Renal and neurological side effects of colistin in critically ill patients. Ann Intensive Care. 2011; 1:14.

4. Lahiry S, Choudhury S, Mukherjee A, Bhunya PK, Bala M. Polymyxin B-Induced diffuse cutaneous hyperpigmentation. J Clin Diagn Res. 2017; 11(2):FD01-FD02.

5. Shih LK, Gaik CL. Polymyxin B induced generalized skin hyperpigmentation in infants. J Pediatr Sci. 2014; 6:e215.

6. Mattos KPH, Lloret GR, Cintra ML, et al. Acquired skin hyperpigmentation following intravenous polymyxin B treatment: a cohort study. Pigment Cell Melanoma Res. 2016; 29(3):388-90.

7. Chang MW. Disorders of hyperpigmentation. En: Bolognia J, Jorizzo J, Schaffer J. Dermatology. Tercera edición. London: Elsevier; 2012. p.1059.

8. Koch-Weser J, Sidel VW, Federman EB, Kanarek P, Finer DC, Eaton AE. Adverse effects of sodium colistimethate: Manifestations and specific reaction rates during 317 courses of therapy. Ann Intern Med. 1970; 72(6):857-68.

9. Chemmangattu R, Jacob S, Pathak HR, Tamilarasi $\mathrm{V}$. Colistin induced neurotoxicity in a patient with end stage kidney disease and recovery with conventional hemodialysis. Open Urol Nephrol J. 2015; 8:53-5.
10. Phe K, Lee Y, McDaneld PM, et al. In vitro assessment and multicenter cohort study of comparative nephrotoxicity rates associated with colistimethate versus polymyxin b therapy. Antimicrob Agents Chemother. 2014; 58(5):2740-6.

11. Deryke CA, Crawford AJ, Uddin N, Wallace MR. Colistin dosing and nephrotoxicity in a large community teaching hospital. Antimicrob Agents Chemother. 2010; 54(10):4503-5.

12. Molina J, Cordero E, Pachon J. New Information about the polymyxin/colistin class of antibiotics. Expert Opin Pharmacother. 2009; 10:2811-2828.

13. Lim LM, Ly N, Anderson D, et al. Resurgence of colistin: a review of resistance, toxicity, pharmacodynamics, and dosing. Pharmacotherapy. 2010; 30(12):1279-91.

Recibido: 10/02/2018

Aceptado: 06/08/2018 